\title{
Chiral symmetry breaking in Hamiltonian QCD in Coulomb gauge
}

\author{
M. Pak and H. Reinhardt \\ Institut für Theoretische Physik, Auf der Morgenstelle 14, 72076 Tübingen, Germany
}

(Dated: November 20, 2018)

\begin{abstract}
Spontaneous breaking of chiral symmetry is investigated in the Hamiltonian approach to QCD in Coulomb gauge. The quark wave functional is determined by the variational principle using an ansatz which goes beyond the commonly used BCS-type of wave functionals and includes the coupling of the quarks to the transversal spatial gluons. Using the lattice gluon propagator as input it is shown that the low energy chiral properties of the quarks, like the quark condensate and the constituent quark mass, are substantially increased by the coupling of the quarks to the spatial gluons. Our results compare favourably with the phenomenological values.
\end{abstract}

Introduction.-The infrared sector of QCD is characterized by two non-perturbative phenomena: confinement and spontaneous breaking of chiral symmetry. For $N_{f}$ massless quark flavours the QCD Lagrangian is invariant under separate global flavour rotations of the leftand right-handed quarks

$$
U_{L}\left(N_{f}\right) \times U_{R}\left(N_{f}\right)=U_{V}\left(N_{f}\right) \times U_{A}\left(N_{f}\right) .
$$

The vector $(V)$ and axial-vector $(A)$ groups rotate leftand right-handed quarks in the, respectively, same and opposite way. In the quantum theory, $U_{A}\left(N_{f}\right)$ is spontaneously broken by quark condensation $\langle\bar{q} q\rangle \neq 0$ resulting in the generation of a constituent quark mass and the occurence of $N_{f}^{2}$ Goldstone bosons. The latter can be identified with the (light) pseudoscalar mesons. Chiral symmetry is a good starting point for the $N_{f}=3$ light quark flavours $u, d, s$. The finite (current) quark masses explicitly break the axial group $U_{A}\left(N_{f}\right)$ and induce finite masses for the pseudoscalar mesons.

Originally the dynamical breaking of chiral symmetry was studied within QCD-inspired models which share with QCD the property of chiral symmetry, e.g., the Nambu-Jona-Lasinio model [1-4] (which, however, is not confining), or models with a confining two-body interaction [5-7]. In recent years there have been also attempts to study chiral symmetry breaking in continuum QCD either by means of Dyson-Schwinger equations [8, [9] or by the renormalization group flow equations [10]. In this letter we study the spontaneous breaking of chiral symmetry within a variational approach, which is the extension to full QCD of the Hamiltonian approach to YangMills theory in Coulomb gauge developed in recent years [1] 14].

Variational calculations in Coulomb gauge Yang-Mills theory were started in Ref. [15] and later on taken up in Ref. 16]. Our approach [11 14] differs from that of Refs. [15, 16] in the ansatz for the vacuum wave functionals and, more importantly, in the full inclusion of the Faddeev-Popov determinant and in the renormalization, for more details see Ref. [17]. Our approach has given a quite satisfactory description of the essential infrared properties of Yang-Mills theory: a linearly rising static quark potential [13], a gluon propagator in accord with
Gribov's formula and with lattice data [18], a perimeter law in the 't Hooft loop 14] and a dielectric function of the Yang-Mills vacuum [19] embodying the phenomenological picture of the bag model. Similar infrared properties were also obtained recently in a renormalization group flow equation approach to Hamiltonian Yang-Mills theory in Coulomb gauge [20].

In the present letter we extend the variational approach in Coulomb gauge to full QCD. We use an ansatz for the vacuum wave functional of the quark sector which goes beyond the BCS-type wave functional used e.g. in Refs. [5, 7] and includes the coupling of the quarks to the transversal spatial gluons [21]. We will show that, using either the lattice results or the results of our variational approach for the gluon sector, this coupling changes the chiral properties of the quarks, like the quark condensate and constitutent mass, by $20-60 \%$ and brings these quantities in the region of their phenomenological values.

Hamiltonian approach to QCD in Coulomb gauge.The part of the QCD Hamiltonian in Coulomb gauge which contains the quark fields $\psi(\boldsymbol{x})$, is given by [24]

$$
H=H_{\mathrm{D}}+H_{\mathrm{QGC}}+H_{\mathrm{C}},
$$

with

$$
\begin{aligned}
H_{\mathrm{D}}= & -i \int d^{3} x \psi^{\dagger}(\boldsymbol{x})\left[\boldsymbol{\alpha} \cdot \boldsymbol{\partial}+\beta m_{0}\right] \psi(\boldsymbol{x}), \\
H_{\mathrm{QGC}}= & -g \int d^{3} x \psi^{\dagger}(\boldsymbol{x}) \boldsymbol{\alpha} \cdot \boldsymbol{A}(\boldsymbol{x}) \psi(\boldsymbol{x}), \\
H_{\mathrm{C}}= & \frac{g^{2}}{2} \int d^{3} x d^{3} y \rho^{a}(\boldsymbol{x}) \hat{F}^{a b}(\boldsymbol{x}, \boldsymbol{y}) \rho^{b}(\boldsymbol{y}) \\
& +\frac{g^{2}}{2} \int d^{3} x d^{3} y \mathcal{J}^{-1} \rho_{\mathrm{dyn}}^{a}(\boldsymbol{x}) \mathcal{J} \hat{F}^{a b}(\boldsymbol{x}, \boldsymbol{y}) \rho^{b}(\boldsymbol{y}) \\
& +\frac{g^{2}}{2} \int d^{3} x d^{3} y \rho^{a}(\boldsymbol{x}) \hat{F}^{a b}(\boldsymbol{x}, \boldsymbol{y}) \rho_{\mathrm{dyn}}^{b}(\boldsymbol{y}) .
\end{aligned}
$$

Here $\boldsymbol{\alpha}$ and $\beta$ are the usual Dirac matrices, $m_{0}$ denotes the current quark mass, and $\boldsymbol{A}(\boldsymbol{x})=\boldsymbol{A}^{a}(\boldsymbol{x}) T^{a}$ is the transversal spatial gauge field in the fundamental representation $T^{a}$ of the gauge group. Furthermore,

$$
\begin{aligned}
\rho^{a}(\boldsymbol{x}) & =\psi^{\dagger}(\boldsymbol{x}) T^{a} \psi(\boldsymbol{x}) \\
\rho_{\mathrm{dyn}}^{a}(\boldsymbol{x}) & =f^{a b c} A_{i}^{b}(\boldsymbol{x}) \Pi_{i}^{c}(\boldsymbol{x})
\end{aligned}
$$


are the quark and gluon color charge densities, respectively, with $f^{a b c}$ being the structure constants of the gauge group and $\Pi_{k}^{a}(\boldsymbol{x})=\delta / i \delta A_{k}^{a}(\boldsymbol{x})$ being the canonical momentum operator of the gauge field. Finally, $\mathcal{J}(A)=\operatorname{Det}(-\hat{D} \partial)$ is the Fadeev-Popov determinant and

$$
\hat{F}^{a b}(\boldsymbol{x}, \boldsymbol{y})=\left\langle\boldsymbol{x}\left|\left[(-\hat{D} \partial)^{-1}\left(-\partial^{2}\right)(-\hat{D} \partial)^{-1}\right]^{a b}\right| \boldsymbol{y}\right\rangle
$$

is the so-called Coulomb kernel where $\hat{D}_{k}^{a b}=\delta^{a b} \partial_{k}+$ $g f^{a c b} A_{k}^{c}$ is the covariant derivative in the adjoint representation.

The Coulomb term $H_{\mathrm{C}}$, Eq. (5), arises from the resolution of Gauss's law, so that gauge invariance is fully taken into account. The Yang-Mills vacuum expectation value of the Coulomb kernel

$$
\left\langle\hat{F}^{a b}(\boldsymbol{x}, \boldsymbol{y})\right\rangle_{\mathrm{YM}}=\delta^{a b} F(\boldsymbol{x}, \boldsymbol{y})
$$

defines the static non-Abelian color Coulomb potential $F(\boldsymbol{x}, \boldsymbol{y})$. Lattice measurements show that this quantity rises linearly at large distances, however, with a coefficient $\sigma_{\mathrm{C}}$ (referred to as Coulomb string tension) which is about two to three times the value of the Wilsonian string tension $\sigma_{\mathrm{w}}[25$ 28]. In momentum space

$$
g^{2} F(k) \rightarrow \frac{8 \pi \sigma_{\mathrm{C}}}{k^{4}}, \quad k \rightarrow 0
$$

Due to asymptotic freedom at small distances the static non-Abelian color Coulomb potential $F(\boldsymbol{x}, \boldsymbol{y})$ behaves essentially like an ordinary Coulomb potential (up to anomalous dimensions).

Variational approach to the quark sector of QCD.We assume the QCD vacuum wave functional to be of the form $|\Phi\rangle=|\phi\rangle \otimes|\mathrm{YM}\rangle$ with a Gaussian type-of wave functional for the Yang-Mills sector [11, 12]

$$
\langle A \mid \mathrm{YM}\rangle=\mathcal{J}^{-\frac{1}{2}}(A) \exp \left(-\frac{1}{2} \int A_{i}^{a}(\boldsymbol{x}) \omega(\boldsymbol{x}, \boldsymbol{y}) A_{i}^{a}(\boldsymbol{y})\right)
$$

For the quark sector we use the following trial ansatz

$$
|\phi\rangle=\mathcal{N} \exp \left(-\int \psi_{+}^{\dagger}(\boldsymbol{x}) K(\boldsymbol{x}, \boldsymbol{y}) \psi_{-}(\boldsymbol{y})\right)|0\rangle,
$$

where $\psi_{ \pm}(\boldsymbol{x})$ are the positive (negative) energy components of the quark field $\psi(\boldsymbol{x})$ and $|0\rangle$ is the vacuum wave functional of the free quarks defined by

$$
\psi_{+}(\boldsymbol{x})|0\rangle=0=\psi_{-}^{\dagger}(\boldsymbol{x})|0\rangle
$$

With arbitrary kernel $K$ the state $|\phi\rangle$ is the most general Slater determinant which is not orthogonal to the vacuum $|0\rangle$. For the kernel $K$ we assume the form

$$
K(\boldsymbol{x}, \boldsymbol{y})=K_{0}(\boldsymbol{x}, \boldsymbol{y})+\int d^{3} z \mathcal{K}(\boldsymbol{x}, \boldsymbol{y} ; \boldsymbol{z}) \cdot \boldsymbol{A}(\boldsymbol{z}),
$$

where $K_{0}$ and $\mathcal{K}$ are determined by minimizing the energy density of the quarks. Finally, $\mathcal{N}$ is a normalization constant, which is a functional of the kernel $K$, Eq. (14), and thus of the gauge field. Through the vector kernel $\mathcal{K}$, the wave functional (12) contains the coupling of the quarks to the gluons. If this coupling is neglected (by putting $\mathcal{K}=0$ ) the wave functional $|\phi\rangle$, Eq. (14), is of the BCS-type used in Refs. [5, 7], for which the expectation value of the quark-gluon coupling term $H_{\mathrm{QGC}}$, Eq. (4), vanishes.

Our strategy is as follows: We first take the expectation value of the quark Hamiltonian H, Eq. (2), in the fermionic state $|\phi\rangle$, Eq. (12), and subsequently in the Yang-Mills vacuum state (11). The fermionic expectation value $\langle H\rangle_{\mathrm{F}}=\langle\phi|H| \phi\rangle$ can be taken exactly (by using Wick's theorem) since the wave functional $|\phi\rangle$ is a Slater determinant. It can be expressed in terms of the static quark propagator (in the presence of the gauge field)

$$
G\left(\boldsymbol{x}, \boldsymbol{x}^{\prime}\right)=\frac{1}{2}\left\langle\phi\left|\left[\psi(\boldsymbol{x}), \psi^{\dagger}\left(\boldsymbol{x}^{\prime}\right)\right]\right| \phi\right\rangle,
$$

which for the state (12) is given in a matrix notation by

$$
\begin{aligned}
G & =\Lambda_{+}\left(1+K K^{\dagger}\right)^{-1} \Lambda_{+}+\Lambda_{-}\left(1+K^{\dagger} K\right)^{-1} K^{\dagger} \Lambda_{+}+ \\
& +\Lambda_{+}\left(1+K K^{\dagger}\right)^{-1} K \Lambda_{-}-\Lambda_{-}\left(1+K^{\dagger} K\right)^{-1} \Lambda_{-}+ \\
& +\frac{1}{2}\left(\Lambda_{-}-\Lambda_{+}\right)
\end{aligned}
$$

where $\Lambda_{ \pm}$are the projectors on positive and negative energy states $\psi_{ \pm}=\Lambda_{ \pm} \psi$. Note that the quark propagator (and accordingly $\langle H\rangle_{\mathrm{F}}$ ) is via the kernel $K$, Eq. (14), a non-local and non-linear functional of the spatial gauge field $\boldsymbol{A}$.

For the gluonic expectation value $\langle\ldots\rangle_{\mathrm{YM}}=$ $\langle\mathrm{YM}|\ldots| \mathrm{YM}\rangle$ of $\langle H\rangle_{\mathrm{F}}$ in the Yang-Mills vacuum state (11), we will restrict ourselves to two (overlapping) loops. To this order, in the gluonic expectation value of $\langle H\rangle_{\mathrm{F}}$ we can use the replacement

$$
\left\langle\ldots\left(1+K^{\dagger} K\right)^{-1} \ldots\right\rangle_{\mathrm{YM}} \rightarrow\left\langle\ldots\left(1+\left\langle K^{\dagger} K\right\rangle_{\mathrm{YM}}\right)^{-1} \ldots\right\rangle_{\mathrm{YM}}
$$

and furthermore, replace the Coulomb kernel (8) by its vacuum expectation value, i.e. by the static non-Abelian color Coulomb potential (9). It is then straightforward, although quite involved, to evaluate $\left\langle\langle H\rangle_{\mathrm{F}}\right\rangle_{\mathrm{YM}}$ and take the variation with respect to the kernels $K_{0}, \mathcal{K}$ and $\omega$. In the present study we ignore the back-reaction of the gluon sector to the presence of the quarks, i.e. we keep the gluonic kernel (the gluon quasi-particle energy) $\omega$ at its form determined in the pure Yang-Mills sector [11 13]. We also ignore the small current quark mass $m_{0}$, which can be easily included if desired. Furthermore, inspired by the form of the quark Hamiltonian $H$, Eq. (2), we assume for the quark kernels (14) of the wave functional (12) the form

$$
\begin{aligned}
K_{0}(\boldsymbol{x}, \boldsymbol{y}) & =\beta S(\boldsymbol{x}-\boldsymbol{y}) \\
\mathcal{K}(\boldsymbol{x}, \boldsymbol{y} ; \boldsymbol{z}) & =\boldsymbol{\alpha} V(\boldsymbol{x}-\boldsymbol{y}, \boldsymbol{z}-\boldsymbol{y}),
\end{aligned}
$$




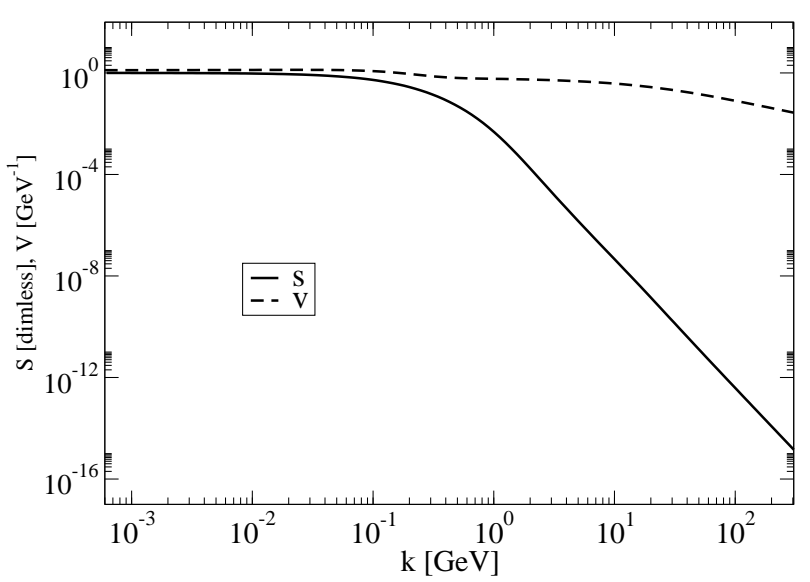

FIG. 1. The variational kernels $S$ and $V$ for $\sigma_{\mathrm{C}}=2 \sigma_{\mathrm{w}}$.

where $S(\boldsymbol{x}-\boldsymbol{y})$ and $V(\boldsymbol{x}-\boldsymbol{y}, \boldsymbol{z}-\boldsymbol{y})$ are scalar functions. In principle, more complex tensor structures of the quarkgluon coupling can be included as has been done in threedimensional QED in Landau gauge [29].

Variation of the energy $\left\langle\langle H\rangle_{\mathrm{F}}\right\rangle_{\mathrm{YM}}$ with respect to the kernels Eq. (18) $S, V$ yields the following gap equations in momentum space

$$
\begin{aligned}
S(\boldsymbol{k}) & =\frac{I_{\mathrm{C}}^{(1)}(\boldsymbol{k})}{|\boldsymbol{k}|-I_{\mathrm{QGC}}(\boldsymbol{k})} \\
V(\boldsymbol{k}, \boldsymbol{p}) & =-\frac{g}{2} \frac{1+S^{2}(\boldsymbol{k})+R(\boldsymbol{k})}{|\boldsymbol{k}|-I_{\mathrm{QGC}}(\boldsymbol{k})+I_{\mathrm{C}}^{(2)}(\boldsymbol{k})},
\end{aligned}
$$

where

$$
R(\boldsymbol{k})=C_{F} \int \frac{d^{3} q}{(2 \pi)^{3}} D(\ell) V^{2}(\boldsymbol{k}, \boldsymbol{q})[1+(\hat{\boldsymbol{k}} \cdot \hat{\boldsymbol{\ell}})(\hat{\boldsymbol{q}} \cdot \hat{\boldsymbol{\ell}})]
$$

with $\boldsymbol{\ell}=\boldsymbol{k}-\boldsymbol{q}, C_{F}=\left(N_{\mathrm{C}}^{2}-1\right) /\left(2 N_{\mathrm{C}}\right)$ being the quadratic Casimir and $D(\ell)$ being the static gluon propagator. In the above equations we have introduced the loop integrals

$$
\begin{aligned}
I_{\mathrm{QGC}}(\boldsymbol{k})= & g C_{F} \int \frac{d^{3} q}{(2 \pi)^{3}} D(\ell) V(\boldsymbol{k}, \boldsymbol{q})[1+(\hat{\boldsymbol{k}} \cdot \hat{\boldsymbol{\ell}})(\hat{\boldsymbol{q}} \cdot \hat{\boldsymbol{\ell}})], \\
I_{\mathrm{C}}^{(1)}(\boldsymbol{k})= & \frac{g^{2}}{2} C_{F} \int \frac{d^{3} q}{(2 \pi)^{3}} \frac{F(\boldsymbol{k}-\boldsymbol{q})}{1+S^{2}(\boldsymbol{q})+R(\boldsymbol{q})} \\
\times & {\left[S(\boldsymbol{q})\left(1-S^{2}(\boldsymbol{k})+R(\boldsymbol{k})\right)+\right.} \\
& \left.-(\hat{\boldsymbol{k}} \cdot \hat{\boldsymbol{q}}) S(\boldsymbol{k})\left(1-S^{2}(\boldsymbol{q})-R(\boldsymbol{q})\right)\right], \\
I_{\mathrm{C}}^{(2)}(\boldsymbol{k})= & \frac{g^{2}}{2} C_{F} \int \frac{d^{3} q}{(2 \pi)^{3}} \frac{F(\boldsymbol{k}-\boldsymbol{q})}{1+S^{2}(\boldsymbol{q})+R(\boldsymbol{q})} \\
\times & {\left[2 S(\boldsymbol{k}) S(\boldsymbol{q})+\frac{1}{2}(\hat{\boldsymbol{k}} \cdot \hat{\boldsymbol{q}})\left(1-S^{2}(\boldsymbol{q})-R(\boldsymbol{q})\right)\right] . }
\end{aligned}
$$

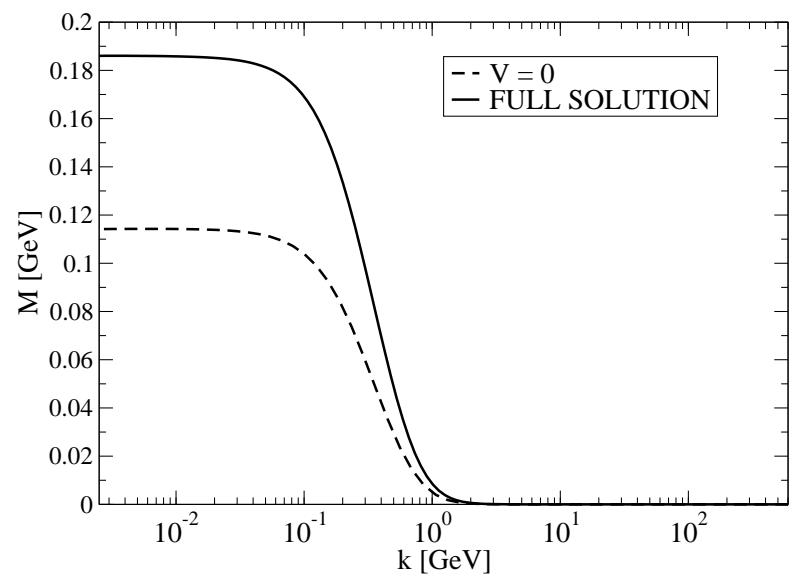

FIG. 2. The dynamical mass $M$ for the full solution and for $V=0$ with $\sigma_{\mathrm{C}}=2 \sigma_{\mathrm{w}}$.

A closer inspection of Eq. (20) shows that the vector kernel $V(\boldsymbol{k}, \boldsymbol{p}) \equiv V(\boldsymbol{k})$ does not depend on the momentum $\boldsymbol{p}$ which is conjugate to the coordinate $\boldsymbol{z}-\boldsymbol{y}$ in (18). After taking the gluonic expectation value of the quark propagator (16) one finds the quark condensate

$$
\langle\bar{\psi} \psi\rangle=-\frac{2 N_{C}}{\pi^{2}} \int d q q^{2} \frac{S(q)}{1+S^{2}(q)+R(q)}
$$

and the dynamical quark mass

$$
M(k)=k \frac{2 S(k)}{1-S^{2}(k)-R(k)} .
$$

Numerical Results.-For the static gluon propagator $D(q)=1 /(2 \omega(q))$ we can take either the result of the variational calculation [11 13 ] or the lattice result [18]. Both propagators are very similar to each other and can be well fitted by Gribov's formula [30] $\omega(k)=$ $\sqrt{k^{2}+M^{4} / k^{2}}$ with $M \approx 880 \mathrm{MeV}$.

The quark Hamiltonian $H$, Eq. (2), like the full QCD Hamiltonian, is scale invariant. In the present case the scale is set by the Coulomb string tension $\sigma_{\mathrm{C}}$, which enters the static quark potential $F$, Eq. (10). Furthermore, the Hamiltonian $H$ contains the coupling constant $g$ explicitly. In principle, after full renormalization the coupling constant should enter only in the form of the running one. Since we are interested here in the infrared sector of QCD we will replace the running coupling calculated in the Hamiltonian approach in Ref. [31] by its (finite) infrared limit. Furthermore, we use $\sigma_{\mathrm{W}}=(440 \mathrm{MeV})^{2}$ and $N_{\mathrm{C}}=3$. With this input the coupled equations (19) and (20) are solved numerically and the results are shown in Figs. 1 and 2. The quark condensate (25) and the dynamical mass (26) are affected in two ways by the inclusion of the coupling of 
the quarks to the spatial gluons: first through the change of $S(\boldsymbol{k})$ and secondly through the occurence of the vector form factor $V(\boldsymbol{k})$ in the coupled equations (19) and (20). Neglecting the coupling of the quarks to the gluons $(\mathcal{K}=0)$ we obtain for the quark condensate

$$
\langle\bar{\psi} \psi\rangle \approx\left(-113 \mathrm{MeV} \sqrt{\sigma_{\mathrm{C}} / \sigma_{\mathrm{w}}}\right)^{3}
$$

which agrees with the findings of Ref. [7] if the same value for the string tension is used. With the inclusion of the quark-gluon coupling this value is shifted to

$$
\langle\bar{\psi} \psi\rangle \approx\left(-135 \mathrm{MeV} \sqrt{\sigma_{\mathrm{C}} / \sigma_{\mathrm{w}}}\right)^{3}
$$

which is a $20 \%$ increase of the figure in the bracket. Lattice calculations show that $\sigma_{\mathrm{C}}=(2 \ldots 3) \sigma_{\mathrm{W}}$, which yields for the quark condensate (28)

$$
\langle\bar{\psi} \psi\rangle \approx(-(191 \ldots 234) \mathrm{MeV})^{3},
$$

which compares favourably with the phenomenological value $\langle\bar{\psi} \psi\rangle=(-230 \mathrm{MeV})^{3}$. An even larger effect of the quark-gluon coupling is obtained for the constituent mass. Neglecting the quark-gluon coupling $(\mathcal{K}=0)$ yields

$$
M \approx 84 \mathrm{MeV} \sqrt{\sigma_{\mathrm{C}} / \sigma_{\mathrm{W}}},
$$

while with this coupling included one finds

$$
M \approx 132 \mathrm{MeV} \sqrt{\sigma_{\mathrm{C}} / \sigma_{\mathrm{W}}},
$$

which is an increase of $57 \%$. Using again the values for $\sigma_{\mathrm{C}}$ quoted above we obtain

$$
M \approx(186 \ldots 230) \mathrm{MeV} .
$$

Conclusions.-Our results show that the coupling of the quarks to the spatial gluons, neglected in previous investigations 5 7], quite substantially influences the infrared chiral properties of the quarks. Typically the effect of the quark-gluon coupling is in the range between $20-60 \%$. To make more accurate predictions within our approach, a more precise lattice measurement of the Coulomb string tension $\sigma_{\mathrm{C}}$ is required, which sets the scale in our approach.

Acknowledgments.-The authors acknowledge useful discussions with G. Burgio, D. Campagnari, M. Leder, M. Quandt and P. Watson. This work was supported by the Deutsche Forschungsgemeinschaft (DFG) under contract Re856/6-3, by the Europäisches Graduiertenkolleg "Hadronen im Vakuum, Kernen und Sternen" BaselGraz-Tübingen and by the Graduiertenkolleg "KeplerKolleg: Particles, Fields and Messengers of the Universe".

[1] Y. Nambu and G. Jona-Lasinio, Phys. Rev. 122 (1961) 345; Y. Nambu and G. Jona-Lasinio, Phys. Rev. 124 (1961) 246.
[2] D. Ebert and H. Reinhardt, Nucl. Phys. B 271 (1986) 188.

[3] D. Ebert, H. Reinhardt and M. K. Volkov, Prog. Part. Nucl. Phys. 33 (1994) 1.

[4] S. P. Klevansky, Rev. Mod. Phys. 64 (1992) 649.

[5] J. R. Finger and J. E. Mandula, Nucl. Phys. B 199 (1982) 168.

[6] A. Le Yaouanc, L. Oliver, O. Pene, J. C. Raynal, Phys. Rev. D29 (1984) 1233.

[7] S. L. Adler and A. C. Davis, Nucl. Phys. B 244 (1984) 469.

[8] A. C. Aguilar and J. Papavassiliou, Phys. Rev. D 83 (2011) 014013.

[9] C. S. Fischer, J. Phys. G 32 (2006) R253.

[10] H. Gies and C. Wetterich, Phys. Rev. D 65 (2002) 065001; H. Gies and C. Wetterich, Phys. Rev. D 69 (2004) 025001.

[11] C. Feuchter and H. Reinhardt, Phys. Rev. D 70 (2004) 105021.

[12] H. Reinhardt and C. Feuchter, Phys. Rev. D 71, (2005) 105002.

[13] D. Epple, H. Reinhardt and W. Schleifenbaum, Phys. Rev. D 75, (2007) 045011.

[14] H. Reinhardt and D. Epple, Phys. Rev. D 76, (2007) 065015.

[15] D. Schutte, Phys. Rev. D 31 (1985) 810; P. Besting, D. Schutte, Phys. Rev. D40 (1989) 2692-2696.

[16] A. P. Szczepaniak and E. S. Swanson, Phys. Rev. D 65, (2002) 025012.

[17] J. Greensite, H. Matevosyan, S. Olejnik, M. Quandt, H. Reinhardt and A. P. Szczepaniak, Phys. Rev. D 83 (2011) 114509.

[18] G. Burgio, M. Quandt and H. Reinhardt, Phys. Rev. Lett. 102, (2009) 032002.

[19] H. Reinhardt, Phys. Rev. Lett. 101 (2008) 061602.

[20] M. Leder, J. M. Pawlowski, H. Reinhardt and A. Weber, Phys. Rev. D 83 (2011) 025010.

[21] In [22] an attempt has been made to include the quarkgluon coupling by means of the "exponential-S" method [23]. However, the underlying eigenvalue problem was not solved.

[22] A. P. Szczepaniak, P. Krupinski, Phys. Rev. D66 (2002) 096006.

[23] H. Kummel, K. H. Luhrmann, J. G. Zabolitzky, Phys. Rept. 36 (1978) 1-36.

[24] N. H. Christ, T. D. Lee, Phys. Rev. D22 (1980) 939.

[25] J. Greensite, S. Olejnik and D. Zwanziger, Phys. Rev. D 69 (2004) 074506.

[26] K. Langfeld and L. Moyaerts, Phys. Rev. D 70 (2004) 074507.

[27] A. Voigt, E. M. Ilgenfritz, M. Muller-Preussker and A. Sternbeck, Phys. Rev. D 78 (2008) 014501.

[28] Y. Nakagawa, A. Nakamura, T. Saito and H. Toki, Phys. Rev. D 83 (2011) 114503.

[29] A. Kizilersu and M. R. Pennington, Phys. Rev. D 79 (2009) 125020.

[30] V. N. Gribov, Nucl. Phys. B 139, (1978) 1.

[31] W. Schleifenbaum, M. Leder and H. Reinhardt, Phys. Rev. D 73 (2006) 125019. 